\title{
Diagnóstico primário dos fatores de risco coronariano em idosos vinculados à estratégia saúde da família no interior do Rio Grande do Sul
}

\author{
Primary diagnosis of coronary risk factors in elderly linked to the \\ family health strategy of Rio Grande do Sul state
}

Micheli Beatriz Radtke, ${ }^{1}$ Francisca Maria Assmann Wichmann, ${ }^{1}$ Analie Nunes Couto' 'Universidade de Santa Cruz do Sul (Unisc), Santa Cruz do Sul, RS, Brasil.

Recebido em: 17/08/2016 / Aceito em: 27/09/2016 / Publicado em: 18/10/2016

micheliradtke@hotmail.com

\section{RESUMO}

Objetivo: estimar a prevalência de fatores de risco de doença coronariana em idosos de um município no interior do Rio Grande do Sul. Método: para a realização do presente estudo, utilizou-se a tabela de risco coronariano proposta pela Michigan Heart Association. Realizou-se um estudo com abordagem epidemiológica, observacional e com delineamento transversal em uma amostra calculada de 115 idosos, de ambos os sexos, na faixa etária média de $69,86 \pm 7,7$ anos. Os dados foram submetidos à análise descritiva, através da média, desvio padrão e identificação do percentual da ocorrência do risco relativo, além disso, foi realizado o teste Mann-Whitney e o teste $t$ pareado para comparação do risco coronariano entre as unidades de saúde e a frequência na dieta alimentar de alimentos ricos em gorduras saturadas e sódio. Resultados: o risco coronariano médio obtido foi de 31,23 \pm 5,3 pontos, classificado pela Michigan Heart Association, como risco moderado. Não houve alteração significativa entre os sexos e as localidades, indicando que o risco coronariano é alto em todo território avaliado. A população apresentou dieta aterogênica, com consumo elevado de gordura saturada e sódio. De acordo com a ordem de prevalência, os fatores mais encontrados foram: percentual de gordura saturada na dieta, hereditariedade, sobrepeso, pressão arterial, sedentarismo e tabagismo respectivamente. Considerações finais: foram evidenciados casos de alto risco, alertando para a necessidade da elaboração e inserção de políticas de promoção de saúde, a fim de minimizar as possibilidades de ocorrência de eventos coronarianos, sobretudo em uma população idosa.
Palavras-chave: Doença da artéria coronariana; Idosos; Promoção da Saúde.

\section{ABSTRACT}

Objective: to estimate the prevalence of coronary heart disease risk factors in elderly in a county located in the interior of Rio Grande do Sul. Method: to carry out this study was used a coronary risk table proposed by the Michigan Heart Association. We conducted a study with epidemiological approach, observational and cross-sectional design in a calculated sample of 115 elderly of both genders. Data were submitted to descriptive analysis, using the mean, standard deviation and identifying the relative risk of occurrence percentage, in addition, it performed the Mann-Whitney test and the paired $t$ test for comparison of coronary risk among health units and the frequency in the diet of foods rich in saturated fats and sodium. Results: we evaluated 115 elderly, the mean age of $69.86 \pm 7.7$ years. The average coronary risk obtained was $31.23 \pm 5.3$ points, classified by Michigan Heart Association as moderate risk. There was no significant change between the sexes and localities, indicating that the coronary risk is high throughout the rated territory. The population showed atherogenic diet with high intake of saturated fat and sodium. Risk factors that were prevalent in the questionnaire responses were: percentage of saturated fat in the diet, heredity, overweight, high blood pressure, sedentary lifestyle and smoking respectively. Closing remarks: it was shown in high-risk cases, warning of the need for development and insertion of health promotion policies in order to 
minimize the chances of occurrence of coronary events, especially in the elderly people.

Keywords: Coronary artery disease; Elderly; Health Promotion.

\section{INTRODUÇÃO}

O crescimento da população idosa é um acontecimento mundial e tem ocorrido de forma acelerada no Brasil, o que tem gerado aumento na demanda dos serviços de saúde. O envelhecimento é considerado um dos grandes desafios. Em todos os países e, especialmente nos países em desenvolvimento, como o Brasil, medidas para ajudar pessoas mais velhas a se manterem saudáveis e ativas são uma necessidade.

Atualmente, as doenças cardiovasculares (DCV) respondem como uma das principais causas de morbidade, mortalidade e gastos hospitalares nos países do mundo ocidental. Os fatores de risco para o desenvolvimento das doenças cardiovasculares estão aumentando progressivamente com a população idosa, e essa situação poderá refletir nos orçamentos de países ricos e pobres. ${ }^{2}$

Para amenizar essa situação, dever-se-ia adotar o conceito de tratamento preventivo de problemas de saúde de longo prazo. O sedentarismo, associado à má alimentação leva à doenças que se tornam fatores de risco para o desenvolvimento de doenças cardiovasculares, como por exemplo, o diabetes mellitus, a hipertensão arterial e a obesidade. ${ }^{3}$

Os fatores de risco cardiovasculares podem ser divididos em riscos modificáveis e não modificáveis, considerando como fatores de risco: as características bioquímicas ou fisiológicas dos indivíduos, associados aos estilos de vida (tabaco, dieta, álcool, sedentarismo) e outros fatores modificáveis. ${ }^{4}$ Esta divisão surge como um conceito moderno que combina o conceito clássico de causa direta de doença, com conceitos mais recentes de probabilidade do desenvolvimento, prevenção e prognóstico. ${ }^{5}$

Estudos epidemiológicos vêm demonstrando forte correlação com os fatores de risco cardiovascular e a razão cintura/estatura (RCEst). Esta tem sido bem aceita como indicador da distribuição da gordura corporal na população, principalmente por ser de fácil obtenção, e com grande utilidade na saúde pública. ${ }^{6}$ Pesquisas realizadas em todo mundo demonstram, a relevância da investigação dos fatores de risco para doenças cardiovasculares. Michigan Heart Association (MHA) adaptou questionário de risco coronariano, que visa classificar a probabilidade de um indivíduo em adquirir doenças cardiovasculares. ${ }^{3,7}$

Como um processo dinâmico e progressivo, ao longo do envelhecimento ocorre alterações morfológicas, funcionais, bioquímicas e psicológicas. Sua dimensão vai além do contexto biológico, e requer, portanto, atenção especial. ${ }^{2}$ Justifica-se, portanto, a necessidade de avaliar periodicamente os idosos, no intuito de detectar problemas de saúde e fatores de risco relacionados com as alterações cardiovasculares. O presente estudo teve como propósito estimar a prevalência de fatores de risco de doença coronariana nos idosos de uma cidade do interior do Rio Grande do Sul.

\section{MÉTODO}

Trata-se de um estudo epidemiológico, observacional, com delineamento transversal, por meio de um questionário validado e padronizado aplicado à população idosa de um município rural do interior do Rio Grande do Sul. O estudo foi realizado com idosos de 60 anos ou mais, de ambos os sexos, na estratégia saúde da família da rede municipal. O município de Sinimbu possui duas unidades de saúde da família (Pinhal e Rio Pequeno), com cobertura de $41 \%$ da população.

Devido à falta de cadastro informatizado de todos os atendimentos de consulta ambulatorial, os procedimentos amostrais foram realizados em três estágios:

Alocação proporcional do número total da amostra nas duas estratégias saúde da família (ESF) do município, conforme proporção de idosos residentes em cada unidade.

Para o cálculo amostral, utilizou-se a calculadora de amostras netquest, disponível online (www.netquest.com/br/painel/painelbook-tamanho-panel-brasil. html), definido uma probabilidade $90 \%$ do resultado ser estendido para o total da população do território com uma margem de erro de $8 \%$.

Contato com os coordenadores técnicos de cada uma das duas unidades de saúde do município para identificação, a partir do arquivo ativo, do nome e endereço de usuários com 60 anos ou mais, cadastrados e a partir dessa listagem, realizou-se uma seleção aleatória simples, ou seja, a escolha dos idosos seguiu a agenda de visitas domiciliares dos agentes comunitários às residências ou a vinda deles até a unidade de saúde.

A amostra do estudo constitui 115 idosos, correspondendo a $92,4 \%$ de probabilidade do resultado se estender à população do território estudado, com uma margem de erro de $8 \%$. Estes foram categorizados nos dois territórios, na ESF Pinhal foram 56 idosos e na ESF Rio Pequeno 59 idosos, correspondendo a $90 \%$ de probabilidade de o resultado ser estendido a população idosa destes territórios.

A coleta dos dados foi realizada pela pesquisadora de dezembro de 2014, a março de 2015, com auxilio dos agentes comunitários de saúde previamente treinados para a pesquisa, inclusive em estudo piloto. Os procedimentos adotados foram: tomada de medidas antropométricas (peso, estatura, circunferência da cintura), e entrevista, com questionário padronizado e pretextado, para obtenção de dados de saúde, de estilo de vida, socioeconômicos e demográficos. As variáveis demográficas analisadas foram o sexo, a idade, o estado civil e a escolaridade. Todos os participantes foram orientados sobre os propósitos do estudo e assinaram o termo de consentimento livre e esclarecido.

Para obter o mesmo padrão na avaliação antropométrica, empregou-se o mesmo material na coleta de dados, uma fita antropométrica metálica de $1,5 \mathrm{~m}$, da marca $\mathrm{RMC}^{\circledR}$ Gel Clinico e para aferição do peso corporal, utilizou-se uma balança da marca Cadence ${ }^{\circledR}$, com carga máxima de $150 \mathrm{~kg}$ e precisão de $100 \mathrm{~g}$.

$\mathrm{Na}$ aferição da estatura foi utilizada uma fita métrica, onde o participante ficou de pé, ereto, com os braços estendidos ao longo do corpo, a cabeça erguida, olhando para um ponto fixo na altura dos olhos, os ossos internos dos calcanhares se tocando, bem como 
a parte interna de ambos os joelhos e os pés unidos mostrando um ângulo reto com as pernas.

A circunferência da cintura foi medida com uma fita métrica inelástica. Esta é uma medida utilizada para avaliar de forma aproximada a massa de gordura intra-abdominal. Permite identificar algumas complicações, como as doenças metabólicas crônicas que estão associadas à deposição da gordura abdominal.

No prontuário clínico de cada paciente, foram coletados nome e número de medicamentos, verificação da pressão arterial sistólica e diastólica, exames laboratoriais como glicose, colesterol, triglicerídeos, sendo considerados somente os valores referentes há sessenta dias anteriores da coleta. A classificação dos níveis de pressão arterial foi conforme as recomendações da Sociedade Brasileira de Cardiologia. ${ }^{8}$ A razão cintura-estatura (RCEst) foi calculada dividindo-se o perímetro da cintura $(\mathrm{cm})$, pela estatura $(\mathrm{cm})$ e admitiu-se como ponto de corte para risco de doença cardiovascular, o valor $\geq 0,5$, para ambos os sexos.

A avaliação do estado nutricional foi realizada por meio do índice de massa corporal (IMC). O IMC foi calculado pela divisão do peso (P) pela estatura (E) ao quadrado: $\mathrm{P}(\mathrm{kg}) / \mathrm{E}(\mathrm{m})^{2} .{ }^{9} \mathrm{O}$ estado nutricional dos idosos foi determinado, segundo pontos de corte recomendados pela Organização Pan-Americana de Saúde: baixo peso (IMC $<23 \mathrm{~kg} / \mathrm{m}^{2}$ ), peso adequado (IMC $\geq 23$ e $\left.\leq 28 \mathrm{~kg} / \mathrm{m}^{2}\right)$, excesso de peso (IMC $>28 \mathrm{e} \leq 30 \mathrm{~kg} / \mathrm{m}^{2}$ ) e obesidade (IMC $\left.>30 \mathrm{~kg} / \mathrm{m}^{2}\right) .^{10}$

Foi aplicado um questionário semiestruturado, com dados sobre o padrão alimentar. Para isso foi aplicado o Questionário adaptado do teste: “Como está a sua alimentação?" do Guia Alimentar de Bolso do Ministério da Saúde. Foram avaliados alimentos fonte de carboidratos, gordura saturada e sódio e os considerados protetores (frutas e verduras). ${ }^{11}$

Para avaliar o risco coronariano relativo, utilizou-se o questionário de risco coronariano proposto pela Michigan Heart Association ${ }^{7}$ e utilizado por Moreira e Marins. ${ }^{12}$ Esse questionário é composto por oito fatores de risco, sendo eles idade, hereditariedade, peso corporal, tabagismo, sedentarismo, hipercolesterolemia ou percentual de gordura na dieta, hipertensão arterial e gênero. Cada fator de risco possui seis opções de resposta. Toda resposta equivale a um escore que representa o risco coronariano relativo aquele fator. A soma dos escores obtidos nas respostas dos oito fatores corresponde a uma pontuação, que representa o risco coronário.

A Michigan Heart Association classifica o risco coronariano como: bem abaixo da média (06-11 pontos); abaixo da média (12-17 pontos); médio (18-24 pontos); moderado (25-31 pontos); alto (32-40 pontos); e muito alto (41-62 pontos). A soma dos escores obtidos nas respostas dos oito fatores corresponde a uma pontuação, que representa o risco cardiovascular, realizando-se o cálculo do percentual da ocorrência do risco coronariano e da taxa de prevalência de cada fator de risco.

A estatística empregada constituiu-se da análise descritiva, através da média e desvio-padrão e da identificação do percentual da ocorrência do risco coronariano. Foi realizado o teste Mann-Whitney e o teste $t$ pareado para comparação do risco coronariano entre as unidades de saúde e a frequência na dieta alimentar de alimentos ricos em gorduras saturadas e sódio. Ambos os testes adotaram o nível de significância de $p<0,05$. Os procedimentos atenderam a Resolução 466/12 do Conselho Nacional de Saúde, sendo previamente aprovados pelo Comitê de Ética em Pesquisa em Seres Humanos da UNISC (n³8254114. 5.0000.5343).

\section{RESULTADOS}

A pesquisa contemplou 115 idosos, sendo 63 $(54,8 \%)$ do sexo feminino e $52(45,2 \%)$ do sexo masculino, com idade entre 60 a 89 anos, com média de $69,86 \pm 7,7$ anos. Em relação a faixa etária, $57,7 \%$ encontravam-se no intervalo de $60-70$ anos, $28,7 \%$ entre $71-80$ anos e $15,7 \%$ acima de 80 anos. Quanto aos anos de estudo, $86,1 \%$ estudaram até o quarto ano do ensino fundamental.

No histórico familiar prevaleceu a doença cardiovascular em $75 \%$. No que se refere aos hábitos de vida, $19 \%(n=22)$ dos participantes faziam uso do cigarro, 60,9\% ( $n=70)$ eram praticantes de atividade física diariamente e $27,6 \%(n=32)$ realizavam atividade física três vezes na semana. Em relação à presença de doenças crônicas, 92,2\% $(n=106)$ diabéticos, 58,3\% $(n=67)$ são hipertensos, destes $46,1 \%$ tem a pressão sistólica acima de 160.

Quanto à classificação das medidas antropométrica $51,3 \%(n=59)$ são obesos e $81,7 \%(n=94)$ possuem gordura abdominal. As mulheres apresentaram significativamente maior prevalência de atividade física, tabagismo, colesterol alto e obesidade $(p<0,05)$.

No tocante aos medicamentos constatou-se que $72,2 \%(n=83)$ dos idosos usam algum tipo de medicamento. Quanto ao número de medicamentos a pesquisa revelou que $34,8 \%(n=40)$ usam até três, $27,8 \%$ $(n=32)$ de quatro a seis e $9,6 \%(n=11)$ mais de seis medicamentos. Apenas $28,8 \%(n=32)$ não fazem uso de medicamentos. Entre as classes mais consumidas estão os cardiovasculares $93 \%$, antidiabéticos com $92 \%$, seguidos dos soníferos $83 \%$, antilipêmicos $76 \%$, anti-hipertensivos $74 \%$ dentre eles destaca-se os diuréticos com $66 \%$.

Já, em relação à frequência de alimentos presentes nas refeições diárias dos idosos, constatou-se uma alta prevalência do consumo de gorduras animais e/ou sólidas (40\%) por $43,5 \%$ dos idosos e $41,7 \%$ relataram consumir $30 \%$ de gordura animal e/ou sólida.

Observou-se no estudo que, de forma geral, os idosos consomem qualitativamente uma alimentação, onde prevalece a produção caseira de alimentos, como a presença de feijão e arroz, verduras, legumes e frutas produzidas no local. Entretanto, percebe-se o relato de consumo diário de alimentos com alta concentração de gordura, sódio e açúcar. Em média $87 \%$ dos idosos consomem quatro ou mais fatias de pão caseiro, $80 \%$ tem o hábito de ingerir bolos ou cucas, $76,5 \%$ referem ingerir algum tipo de doce ou sobremesa, $60 \%$ consomem massas de qualquer tipo, $73 \%$ possuem a batata inglesa ou doce ou aipim nas refeições, estes sempre acompanhados por feijão e arroz.

Do mesmo modo em que o alto consumo de carboidratos na população é preocupante, a presença de alimentos com alta concentração de gorduras animais e/ou sólidas, como frituras, óleos, bacon, torresmo, molhos, enlatados e embutidos foi referido por mais de 
$80 \%$ dos idosos como hábito diário. O estudo apresentou uma associação significativa $(p<0,05)$ entre o risco coronariano e o consumo diário dos alimentos referidos, com predomínio de uma dieta aterogênica, com consumo acima do adequado para frações lipídicas e sódio, do mesmo modo em que o estudo revela um consumo de verduras, legumes e frutas na alimentação diária dos idosos, em mais de $70 \%$ da amostra, podendo este ser considerado um fator protetor.

$\mathrm{Na}$ totalidade de indivíduos da amostra, o escore médio de risco coronariano encontrado foi de 31,23 \pm 5,3 pontos, com valores limítrofes entre 21 e 43 pontos. Os idosos que se encontram na faixa etária dos 60 aos 70 anos apresentaram escores de risco coronariano significativamente maior $(p<0,05)$, quando comparados aos idosos que se encontram na faixa etária acima dos 70 anos. $O$ número de medicamentos associou-se significativamente $(p=0,013)$ com o risco coronariano, logo, indica que existe uma tendência na distribuição do uso dos medicamentos com as categorias do risco coronariano.

Nos sujeitos do gênero masculino, encontrou-se um risco médio de 31,77 pontos, com valores limítrofes entre 21 e 43 pontos, para uma faixa etária média de $70 \pm 7,7$ anos (60 a 89 anos). Já, nos sujeitos do gênero feminino foi encontrado um risco médio de 30,78 , com valores extremos entre 21 e 43 pontos, para uma faixa etária média de 71 \pm 8 anos (60 a 89 anos). Em relação às localidades, o risco médio não apresentou alterações significantes ( $p>0,05)$, permanecendo em torno de $31 \pm 4,87$ pontos.

O percentual de classificação de risco coronariano dos avaliados no presente estudo, como observado na figura 1 , apontou que $46 \%$ dos idosos se encontram em alto risco e $40 \%$ em risco moderado. Em referência às localidades, o estudo não observou alteração significantes na prevalência de riscos coronarianos entre os idosos. Na localidade de Pinhal 50\% $(n=30)$ na média apresentam risco médio a moderado para desenvolver doenças coronarianas e $47 \%(n=26)$ alto risco a muito alto, já em Rio Pequeno $49 \%(n=29)$ apresentam risco médio a moderado e $51 \%(n=30)$ alto risco a muito alto para desenvolver doenças coronarianas.

Já, na figura 2, está disposta a prevalência de cada fator de risco coronariano, sendo que, dentre os seis fatores, aqueles que mais estiveram prevalentes, nas respostas dos questionários, foram: percentual de alimentos ricos em gorduras saturada, hereditariedade, excesso de peso, pressão arterial, fumo e sedentarismo respectivamente.

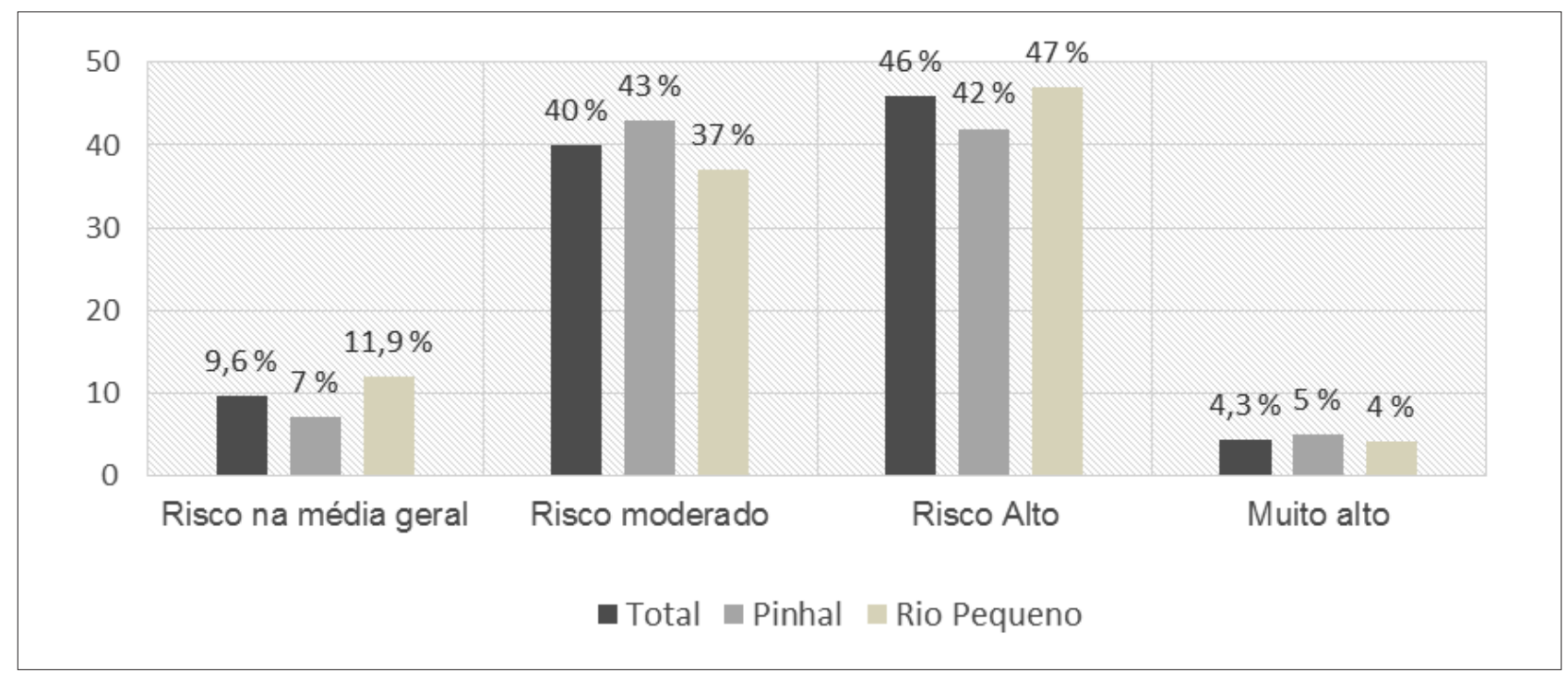

Figura 1 - Frequência da Categorização do Risco Cardíaco no município e nas localidades avaliadas, 2015.

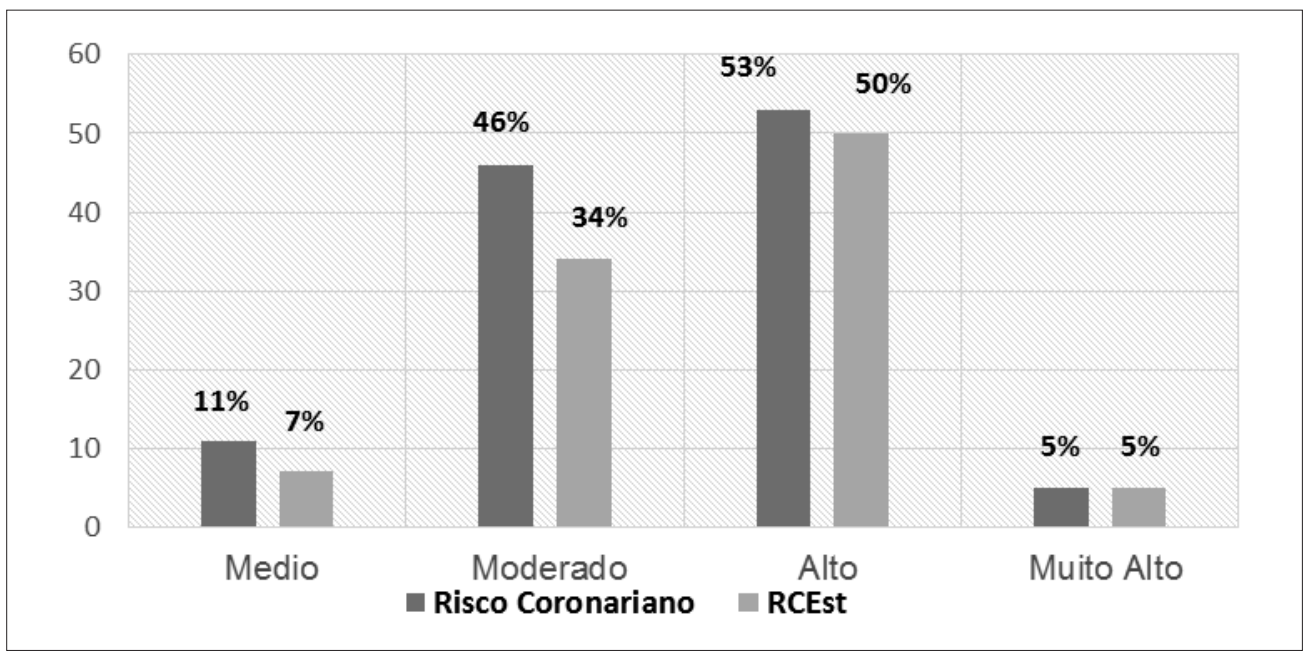

Figura 2 - Prevalência de cada fator de risco coronariano entre os idosos estudados no município de Sinimbu, RS, 2015. 


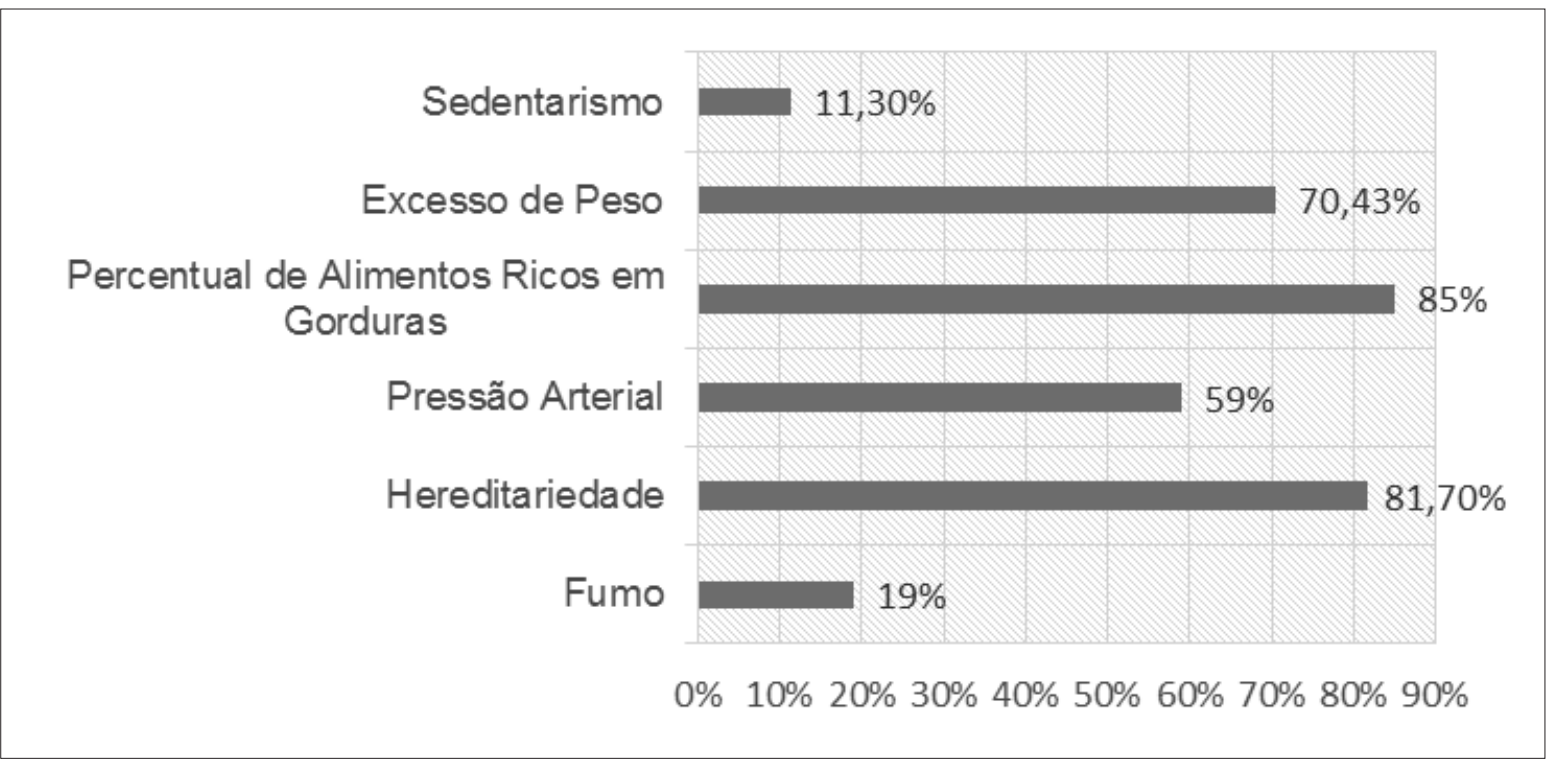

Figura 3 - Prevalência do risco coronariano entre os idosos com a obesidade central identificada pela relação cintura/ estatura, 2015.

Foi observado também um aumento da obesidade central (RCEst $>0,5$ ) em $81,7 \%$ dos idosos. O estudo evidenciou uma associação significante $(p=0,008)$ entre o risco coronariano dos idosos com a obesidade central. Conforme a figura 3 , constatou-se que dos $53 \%$ idosos que apresentam alto risco, $50 \%$ possui concomitantemente obesidade abdominal, indicando uma tendência na distribuição da obesidade central (RCEst) com as categorias do risco coronariano.

\section{DISCUS5ÃO}

Os dados obtidos na população total deste estudo apontam para um risco moderado, com pontuação média de 31,22 ( $\pm 5,18)$, segundo a Michigan Heart Association. Verificou-se maior percentual de idosos do sexo feminino, $63(54,8 \%)$, o que, corrobora com os resultados de outras pesquisas, onde também houve predominância feminina. De acordo com o Censo Demográfico de 2010, as mulheres vivem em média 7,6 anos a mais que os homens; dado este que pode justificar o maior número de mulheres nos estudos realizados com idosos. ${ }^{13,14}$

Em outros estudos que utilizaram o mesmo instrumento, o escore médio de risco coronariano encontrado foi de 22,1 \pm 4,7 pontos, com valores limítrofes entre 10 e 36 pontos, para uma faixa etária média de 43,9 \pm 7,8 anos de idade, com variação de 21 a 58 anos $^{15}$ e em professores da mesma instituição de ensino o escore foi de 22,6 $\pm 4,7$ pontos, para um grupo com idade média de 43,5 $\pm 8,4$ anos. $^{12}$

O percentual de classificação de risco coronariano dos avaliados no presente estudo apontou que, 4,3\% $(n=5)$ tem risco avançado para desenvolver doenças coronarianas, mas não foi constatado nenhum indivíduo na faixa de classificação de risco bem abaixo da média, que seria uma classificação considerada ideal. Os indivíduos compreendidos na faixa etária dos 60 aos 70 anos apresentaram prevalência de risco alto para o desenvol- vimento de DCV, entretanto, os compreendidos na faixa etária acima dos 70 anos, apresentaram os melhores resultados, com prevalências de risco moderado, com o avançar da idade. Essa constatação vai ao encontro dos resultados obtidos por McArdle, Kacth e Katch ${ }^{16}$, os quais afirmam que após os 35 anos ocorre uma tendência de aumento do risco coronariano. Isso torna evidente a influência do fator de risco não modificável idade, como um agravante, na população.

Como se trata de uma população que já possui um risco coronariano alto, este dado incrementa a possibilidade de ocorrência de eventos cardiovasculares e a associação de um ou mais fatores tem um efeito aditivo, aumentando ainda mais esse risco. Entretanto no presente estudo, este dado deve ser avaliado com cautela, pois a amplitude da variação da idade foi elevada.

O histórico familiar de doença cardiovascular foi obtido em $75 \%$ dos participantes. No estudo de Müller et al., ${ }^{17}$ que avaliou os fatores de risco cardiovascular e a qualidade de vida em idosos, foram encontrados $37,5 \%$, dos idosos, com histórico familiar de doenças cardiovasculares, porém esse estudo encontrou um número ainda maior. Dada a alta prevalência dos fatores de risco relacionados à história familiar, reforça-se ainda mais a importância do planejamento de ações que visem a diminuir a prevalência de fatores de risco exógenos, já que estes contribuem com cerca de $75 \%$ com as doenças cardiovasculares. Estudo epidemiológico têm fornecido evidências sobre a importância da dieta como fator de risco para doenças cardiovasculares. ${ }^{18}$

Os fatores de risco mais prevalentes foram: percentual de alimentos ricos em gorduras, hereditariedade, excesso de peso, pressão arterial, fumo e sedentarismo respectivamente. Tendo em vista que os fatores de risco são de caráter modificável, exceto a hereditariedade, é necessária uma intervenção preventiva sobre os mesmos, visando diminuí-los. Tem-se o excesso de peso como o terceiro fator de risco mais prevalente, atingindo $70 \%$ da amostra. A configuração desse quadro advém da correlação de outros fatores, a 
ingesta calórica de alimentos com altas concentrações de gorduras sólidas e/ou líquidas, que coopera para o aumento do tecido adiposo. Vários alimentos e nutrientes têm sido relacionados com a ocorrência de doenças crônicas na população, destacando-se o consumo excessivo de colesterol e gorduras saturadas, nutrientes implicados na gênese das doenças cardiovasculares e a pesquisa revelou que $80 \%$ dos idosos têm como hábito diário o consumo de frituras, óleos, bacon, torresmo, molhos, enlatados e embutidos, que são considerados prejudiciais para a saúde. ${ }^{18}$

Constatou-se nesse estudo que mais da metade $(58,3 \%)$ dos entrevistados apresenta hipertensão arterial e destes $46,1 \%$ tem a pressão sistólica acima de 160. Em indivíduos jovens, a hipertensão decorre mais frequentemente apenas da elevação na pressão diastólica, enquanto a partir da sexta década, o principal componente é a elevação da pressão sistólica. ${ }^{19}$ Resultado semelhante foi encontrado em indivíduos idosos da cidade de Bambuí, onde 61,5\% apresentavam hipertensão arterial. ${ }^{20}$

A relação entre o aumento da pressão arterial e o avanço da idade é maior em populações com alta ingestão de sal e outros alimentos ricos em sódio. Povos que consomem dieta com reduzido conteúdo de sal têm menor prevalência de hipertensão e a pressão arterial não se eleva com a idade. ${ }^{21}$ Os alimentos considerados "de risco", ricos em sódio e gorduras saturadas, por exemplo, devem ser evitados, ao passo que os "de proteção", ricos em fibras e potássio, são permitidos. ${ }^{22}$ Referente aos alimentos de proteção foi encontrado um resultado satisfatório no estudo, pois o consumo de verduras, legumes e frutas se faz presente em mais de $70 \%$ da amostra.

Aproximadamente, $85,2 \%$ idosos consomem mais de $30 \%$ de gordura animal ou sólida, número preocupante, devido que o consumo de gordura saturada e trans está relacionado com elevação do LDL-c plasmático e aumento de risco cardiovascular. A substituição de gordura saturada da dieta por mono e poli-insaturada é considerada uma estratégia importante para o controle da hipercolesterolemia e consequente assim há redução da chance de doenças cardiovasculares. ${ }^{23} \mathrm{De}$ acordo com Pesquisa de Orçamentos Familiares - POF 2008-2009, comparada à mesma pesquisa realizada em 2002-2003, a evolução do consumo de alimentos no domicílio indica aumento de alimentos industrializados. ${ }^{24}$ Porém, nesse estudo, o resultado foi inverso, prevalecendo à produção caseira de alimentos, como a presença de feijão e arroz, verduras, legumes e frutas produzidos no local.

Em relação à distribuição de macronutrientes, o perfil atual mostra que $59 \%$ das calorias estão representadas por carboidratos; $12 \%$, por proteínas; e $29 \%$, por lipídeos. Nas regiões economicamente mais desenvolvidas (Sul, Sudeste e Centro-Oeste do Brasil) e, de modo geral, no meio urbano e entre famílias com maior renda, existe consumo elevado de gorduras, especialmente as saturadas. A mudança na composição nutricional associada à disponibilidade domiciliar de alimentos evidencia diminuição do consumo de carboidratos em detrimento do aumento do teor em gorduras e proteínas. A fração dos carboidratos que mais se reduz é a que exclui os açúcares simples, enquanto a participação das proteí- nas de origem animal aumentou. ${ }^{24}$

Boa parte da população $60,9 \%(n=70)$ pratica atividade física. Realizar atividades como cuidar da horta, jardim, animais domésticos e da casa, deixam esses idosos mais ativos. A tradição em realizar trabalhos no ambiente doméstico é também revelada em estudo com idosos no interior do Rio Grande do Sul, onde metade deles continua realizando trabalhos domiciliares não remunerados. ${ }^{25}$

Outra preocupação é o grande número de diabéticos, em que $92,2 \%(n=106)$ da população estudada possui a doença. O risco de diabete, como por exemplo, o tipo 2, aumenta com a idade e é incrementado por obesidade, sedentarismo e dieta inadequada. Esses fatores que tendem a andar juntos mostraram altas prevalências entre os idosos brasileiros. O diabetes é um grave fator de risco cardiovascular. ${ }^{26} \mathrm{Em}$ um estudo com dos 872 idosos, que compunham a amostra do ISA-capital, foram encontrados $17,6 \%$ de diabéticos ${ }^{27}$, e em dados do Vigitel (2008), encontrou-se 20,7\% pessoas com a enfermidade, ${ }^{28}$ valores baixos quando comparados ao encontrado nesse estudo.

A gordura abdominal foi aferida, estando elevada em $81,9 \%(n=94)$ dos idosos participantes. O risco de doenças cardiovasculares não é determinado somente em relação à quantidade total de gordura do corpo humano, mas principalmente com o tipo de distribuição corporal dessa gordura. ${ }^{29}$ Nesse contexto, o acúmulo de gordura abdominal vem sendo considerado como a que oferece maior risco para a saúde do individuo, sendo relacionada com o aumento do risco de infarto do miocárdio e acidente vascular cerebral. ${ }^{30}$

Para a RCEst, a maioria da população estudada está em risco $(81,7 \%)$, semelhante ao observado por Silva et al. $(96,3 \%) .{ }^{31}$ Chagas et al. ${ }^{32}$ citam a forte correlação da RCEst com a gordura intra-abdominal, e sua associação com maior risco para DCV. Diversos estudos têm demonstrado que a RCEst é um bom discriminador de obesidade abdominal relacionada a fatores de risco cardiovascular, bem como de risco coronariano elevado (RCE). ${ }^{33,34} \mathrm{Em}$ Taiwan, utilizando-se como amostra 38.556 sujeitos de ambos os sexos, observou-se forte associação da RCEst com hipertensão arterial, intolerância à glicose, diabetes e dislipidemias. ${ }^{35}$

O excesso de massa corporal é um fator predisponente para várias doenças. Dos idosos entrevistados $51,3 \%(n=59)$ eram obesos. Resultado semelhante a esse, foi encontrado no estudo intitulado de Prevalência de Sobrepeso e Obesidade em Idosas Atendidas nos Projetos da Universidade Estadual do Sudoeste da Bahia - UESB, das 25 idosos da cidade de Jequié-Bahia participantes, $60 \%$ estavam com sobrepeso e obesidade. ${ }^{36}$ Bahia et al. ${ }^{37}$ estimou que os custos totais por ano (2009 - 2011), com todas as doenças relacionadas ao excesso de peso e à obesidade foram de US\$2,1 bilhões, sendo US\$ 1,4 bilhão $(68,4 \%$ do custo total) devido às internações e os US\$ 679 milhões dólares devido a procedimentos ambulatoriais. Cerca de $10 \%$ destes custos foi atribuído ao excesso de peso e à obesidade.

Um fator protetor na população estudada é o baixo índice de fumantes entre os idosos sendo uma região de produção de tabaco. A baixa prevalência de tabagismo na população estudada pode ser decorrente das campanhas antitabagismo, aumentando a consciência 
a respeito dos males causados pelo cigarro. Os efeitos benéficos da suspensão do fumo são evidentes, em todas as faixas etárias, até mesmo nos idosos, principalmente em termos de qualidade e expectativa de vida. ${ }^{38}$ A prevalência de tabagismo em idosos verificada neste estudo foi semelhante à observada em Porto Alegre/RS, onde apenas $(11,6 \%)$ da população avaliada fumava. ${ }^{39}$

Constatou-se que $72,2 \%$ ingerem algum tipo de medicamento. Quanto ao número de medicamentos a pesquisa revelou que $34,8 \%(n=40)$ ingerem até três, $27,8 \%(n=32)$ de quatro a seis e 9,6\% $(n=11)$ mais de seis medicamentos. Esse resultado é semelhante ao observado em outros estudos realizados em cidades brasileiras, nos quais a prevalência de uso de medicamentos por idosos tem variado entre 70-92\%, com média de utilização entre dois e cinco medicamentos. ${ }^{40,}{ }^{41}$ Uma maior utilização de medicamentos pela população idosa é esperada em função da maior ocorrência de doenças crônicas nesta faixa etária, mas seu consumo elevado sugere a contribuição do valor simbólico do medicamento, que propicia a medicalização, ${ }^{42}$ bem como a baixa frequência de uso de recursos não farmacológicos para o tratamento de doenças. O agravamento dos problemas de saúde juntamente com as doenças crônicas, o envelhecimento da população e a falta de programas de prevenção elevam os gastos com internações e distribuição de medicamentos no sistema público de saúde. ${ }^{43}$

\section{CONSIDERAÇÕES FINAIS}

No presente estudo, a população analisada se encontrava na categoria risco moderado a alto para doença arterial coronariana. O estudo revelou uma associação significativa entre a gordura central e o risco coronariano, incrementando a predição das alterações metabólicas e os comportamentos de risco para desenvolvimento de doenças crônicas. Os valores extremos observados no presente estudo apontam para a necessidade da implantação de um serviço de orientação nutricional, visando melhorar a ingesta de nutrientes, por parte dos indivíduos avaliados.

A identificação desses fatores de risco para doença cardiovascular por meio de estudos de base populacional como este constitui a primeira etapa para elaboração de planos preventivos, os quais contribuíram para uma queda substancial na mortalidade para doenças cardiovasculares, em quase todos os países desenvolvidos. Diante disso, faz-se necessário a continuidade deste estudo, levando-se em consideração a ampliação da amostra, referente ao número de indivíduos e nível sociocultural e econômico.

\section{REFERÊNCIAS}

1. World Health Organization. Preventing chronic diseases: a vital investment. WHO Global Report. Geneva: World Health Organization; 2005.

2. Benedetti TRB, Meurer ST, Morini S. Índices antropométricos relacionados a doenças cardiovasculares e metabólicas em idosos. Ver Educ Fis UEM 2012;23(1):123-30. doi 10.4025/ reveducfis.v23i1.11393.

3. World Health Organization. Expert Consultation on Diet,
Nutrition and the Prevention of Chronic Diseases Diet, nutrition and the prevention of chronic diseases: report of a joint WHO/FAO expert consultation. Geneva: WHO; 2007.

4. Giria, JAA. Carta Europeia para a Saúde do Coração. Boletim Sociedade Portuguesa de Cardiologia 2007;6(135):9-12.

5. Gomes RC. Doenças Cardiovasculares. No Brasil, homens são os mais afetados pelas doenças cardiovasculares, como infarto e AVCs. Portal Brasil, página desenvolvida pelo Ministério da Saúde. Brasil; 2014. Disponível em: http:// www.brasil.gov.br/saude/2011/09/doencas-cardiovasculares

6. Pitanga FJG, Lessa I. Razão cintura-estatura como discriminador do risco coronariano de adultos. Rev Assoc Med Bras 2015;52(3):157-161. doi: 10.1590/S010442302006000300016.

7. Mcardle WD, Katch FI, Katch VL. Fisiologia do exercício: energia, nutrição e desempenho humano. 5 ed. Rio de Janeiro: Guanabara Koogan, 2003.

8. Sposito AC, Caramelli B, Fonseca FA, Bertolami MC, Afiune Neto A, Souza AD, et al.; Sociedade Brasileira de Cardiologia. IV Diretriz brasileira sobre dislipidemias e prevenção da aterosclerose. Arq Bras Cardiol 2007;88(supl 1):1-18.

9. Quetelet LAJ. A treatise on man and the development of his faculties . Obes Res 1994;2(1):72-85. doi: 10.1002/j.15508528.1994.tb00047.x.

10. Organização Pan-Americana. XXXVI Reunión del Comitê Asesor de Ivestigaciones en Salud - Encuestra Multicêntrica - Salud Beinestar y Envejecimeiento (SABE) en América Latina e el Caribe. [online]. Washington, Estados Unidos; 2001.

11. Brasil. Ministério da Saúde. Guia Alimentar de Bolso como ter uma alimentação saudável: dez passos para uma alimentação saudável. Brasília: MS; 2007.

12. Moreira OC, Marins JCB. Estudo do risco coronariano em professores do Centro de Ciências Biológicas e do Centro de Ciências Humanas da Universidade Federal de Viçosa. Coleção Pesquisa em Educação Física 2006;4(1):405-409.

13. Caetano JA, Costa AC, Santos ZMSA, Soares E. Descrição dos fatores de risco para alterações cardiovasculares em um grupo de idosos. Texto Contexto Enferm 2008;17(2):327-35. doi: 10.1590/S0104-07072008000200015

14. Instituto Brasileiro de Geografia e Estatística. Censo demográfico 2010.

15. Moreira OC, Oliveira CEP, Teodoro BG, Souza GC, Lizardo FB, Santos LA, Marins JCB. Fatores de risco de doença cardiovascular em técnicos administrativos da Universidade Federal de Viçosa. Bioscience Journal 2009;25(5):133-40.

16. Mcardle WD, Katch FI, Katch VL. Fisiologia do exercício: energia, nutrição e desempenho humano. Rio de Janeiro: Guanabara Koogan, 2001.

17. Müller CL, Vescovi CC, Santos BRL, Gustavo AS, Creutzberg M, Feoli AMP. Fatores de risco cardiovascular e qualidade de vida de idosos: um estudo preliminar. Rev Graduação 2011;4(1):1-20.

18. Lopes ACS, Caiaffa WT, Sichieri R, Mingoti SA, LimaCosta MF. Consumo de nutrientes em adultos e idosos em estudo de base populacional: Projeto Bambuí. Cad. Saúde Pública 2005;21(4):1201-1209. doi: 10.1590/S0102$311 \times 2005000400022$.

19. Franklin SS, Pio Jr, Wong ND, Larson MG, Leip EP, Vasan RS, Levy D. Predictors of new-onset diastolic and systolic hypertension. The Framinghan Heart Study. Circulation. 2005;111:1121-7. doi: 10.1161/01.CIR.0000157159.39889.EC

20. Barreto SM, Passos VMA, Firmo JOA, Guerra HL, Vidigal PG, Lima-Costa MFF. Hypertension and clustering of 
cardiovascular risk factors in a community in Southeast Brazil: the Bambuí Health and Ageing Study. Arq. Bras. Cardiol 2001;77(6):576-81. doi: 10.1590/S0066$782 \times 2001001200008$.

21. Sociedade Brasileira de Cardiologia. $\vee$ Diretrizes Brasileiras de Hipertensão. Arq Bras Cardiol 2006 Fev: 1-48.

22. Millen BE, Quatromoni PA, Copenhafer DL, Demissie S, O'Horo CE, D'Agostino RB. Validation of a dietary pattern approach for evaluating nutritional risk: the Framingham Nutrition Studies. J Am Diet Assoc 2001;101(2):187-194.

23. Santos RD, Gagliardi ACM, Xavier HT, Magnoni CD, Cassani $\mathrm{R}$, Lottenberg AMP et al. I Diretriz sobre o consumo de gorduras e saúde cardiovascular. Arq. Bras. Cardiol. [Internet] 2013;100(1 Suppl 3):1-40.

24. Instituto Brasileiro de Geografia e Estatística. Pesquisa de Orçamentos Familiares 2008-2009: tabela de medidas referidas para os alimentos consumidos no Brasil. Rio de Janeiro. IBGE; 2011.

25. Morais EP, Rodrigues RAP, Gerhardt TE. Os idosos mais velhos no meio rural: realidade de vida e saúde de uma população do interior gaúcho. Texto Contexto Enferm 2008;17(2):374-83 doi: 10.1590/S0104-07072008000200021.

26. Barreto SM, Passos VMA, Almeida SKF, Assis TD. The burden of diabetes mortality is increasing among Brazilian adults. Rev Panam Salud Publica 2007;22:239-45. doi: 10.1590/S1020-49892007000900003.

27. Mendes $T A B$, Goldbaum M, Segri N, Barros MBAB, Cesar CLG, Carandina L, Alves MCGP. Diabetes mellitus: fatores associados à prevalência em idosos, medidas e práticas de controle e uso dos serviços de saúde em São Paulo, Brasil. Cad Saúde Pública 2011;27:1233-43. doi: 10.1590/S0102 $311 \times 2011000600020$.

28. Ministério da Saúde. Dia mundial do diabetes. Dados estatísticos no Brasil 2009. [online]. Brasília, Brasil; 2009.

29. Rocca SVdS, Tirapegui J, Melo CMd, Ribeiro SML. Efeito do exercício físico nos fatores de risco de doenças crônicas em mulheres obesas. Revista Brasileira de Ciências Farmacêuticas 2008;44:185-92. doi: 10.1590/S151693322008000200004.

30. Pitanga FJG, Lessa I. Indicadores antropométricos de obesidade como instrumento de triagem para risco coronariano elevado em adultos na cidade de Salvador Bahia. Arq. Bras. Cardiol 2005;85(1):26-31. doi: 10.1590/ S0066-782X2005001400006.

31. Silva ARA, Dourado KF, Pereira PB, Lima DSC, Fernandes $A O$, Andrade $A M$, et al. Razão TG/HDL-c e indicadores antropométricos preditores de risco para doença cardiovascular. Rev Bras Cardiol 2012;25(1):41-9.

32. Chagas P, Caramori P, Barcellos C, Galdino TP, Gomes I, Schwanke CHA. Associação de diferentes medidas e índices antropométricos com a carga aterosclerótica coronariana. Arq Bras Cardiol 2011;97(5):397-401.

33. Lin WY, Lee LT, Chen CY, Lo H, Hsia HH, Liu IL, et al. Optimal cut-off values for obesity: using simple anthropometric indices to predict cardiovascular risk factors in Taiwan. Int $\mathrm{J}$ Obes Relat Metab Disord 2002;26(9):1232-8. doi: 10.1038/ sj.ijo.0802040.

34. HoSY, Lam TH, Janus ED. Waist to stature ratio is more strongly associated with cardiovascular risk factors than other simple anthropometric indices. Ann Epidemiol 2003;13(10):683-91. doi: 10.1016/S1047-2797(03)00067-X.

35. Huang KC, Lin WY, Lee LT, Chen CY, Lo H, Hsia HH, Liu $\mathrm{IL}$, Shau WY, Lin RS. Four anthropometric indices and cardiovascular risk factors in Taiwan. Int J Obes Relat Metab Disord 2002;26(8):1060-8. doi: 10.1038/sj.ijo.0802047.

36. Queiroz $\mathrm{CO}$, Munaro HLR. Prevalência de Sobrepeso e Obesidade em Idosas Atendidas nos Projetos da UESB. Rev Saúde.com 2008;4(1):43-9.

37. Bahia L1, Coutinho ES, Barufaldi LA, Abreu Gde A, Malhão TA, de Souza CP, Araujo DV. The costs of overweight and obesity-related diseases in the Brazilian public health system: cross-sectional study. BMC Public Health 2012;12:440. doi: 10.1186/1471-2458-12-440.

38. Goulart D, Engraff P, Ely LS, Sgnaolin V, Santos EF, Terra NL, et al. Tabagismo em idosos. Rev Bras Geriatr Gerontol 2010;13(2):313-20.

39. Paskulin LMG, Vianna LAC. Perfil sociodemográfico e condições de saúde autorreferidas de idosos de Porto Alegre. Rev Saúde Pública 2007;41:757-68. doi: 10.1590/S003489102007000500010.

40. Loyola Filho Al, Uchoa E, Lima-Costa MF. Estudo de base populacional sobre o consumo de medicamentos entre idosos: Projeto Bambuí. Cad Saúde Pública 2005;21:54553. doi: 10.1590/S0102-311X2005000200021.

41. Ribeiro $\mathrm{AQ}$, Rozenfeld $\mathrm{S}$, Klein $\mathrm{CH}$, César $\mathrm{CC}$, Acurcio FA. Inquérito sobre uso de medicamentos por idosos aposentados, Belo Horizonte, MG. Rev Saúde 2008;42:72432. doi: 10.1590/S0034-89102008005000031.

42. Lefévre F. A função simbólica dos medicamentos. Rev Saúde Pública 1983;17:500-3. doi: 10.1590/S003489101983000600007.

43. Flores LM, Mengue SS. Uso de medicamentos por idosos em região do sul do Brasil. Rev Saúde Pública 2005;39:9249. doi: 10.1590/S0034-89102005000600009.

Como citar: RADTKE, Micheli Beatriz; WICHMANN, Francisca Maria Assmann; COUTO, Analie Nunes. Diagnóstico primário dos fatores de risco coronariano em idosos vinculados à estratégia saúde da família no interior do Rio Grande do Sul. Cinergis, Santa Cruz do Sul, v. 17, out. 2016. ISSN 2177-4005. Disponível em: < https://online.unisc.br/seer/index.php/cinergis/ article/view/8152 >. Acesso em: 11 out. 2016. doi:http://dx.doi.org/10.17058/cinergis.v17i0.8152. 